\title{
Diffuse Lewy body disease: clinical features in nine cases without coexistent Alzheimer's disease
}

M A Hely, W G J Reid, G M Halliday, D A McRitchie, J Leicester, R Joffe, W Brooks, G A Broe, J G L Morris

\begin{abstract}
Objective-To further elucidate the relation between diffuse Lewy body disease and Parkinson's disease.

Methods and results-The clinical features of nine cases of pure diffuse Lewy body disease without pathological evidence of coexisting Alzheimer's neuritic pathology are reported. All patients were aged less than 70 years at onset (mean 62 years). Five patients presented with clinical features, which included assymetric resting tremor and levodopa responsiveness, which were initially indistinguishable from idiopathic Parkinson's disease. All five patients later became demented (mean of three years after presentation). Two further patients presented with parkinsonism and dementia and two patients presented with dementia and developed parkinsonism at a later stage. Hallucinations appeared 2.5-9 years after the onset of symptoms in six patients and were a presenting feature in one patient. All patients met the pathological criteria of idiopathic Parkinson's disease, with respect to the midbrain changes, in addition to having diffuse cortical Lewy bodies.

Conclusions-Diffuse Lewy body disease may present as parkinsonism, dementia, or both depending on whether the Lewy body pathology begins in the midbrain, the cortex, or both together. When it begins in the midbrain, diffuse Lewy body disease is indistinguishable initially from idiopathic Parkinson's disease. Diffuse Lewy body disease may be a common cause of dementia complicating Parkinson's disease.
\end{abstract}

( $\mathcal{F}$ Neurol Neurosurg Psychiatry 1996;60:531-538)

Neurology, Royal

Prince Alfred Hospital J Leicester

Department of

Neurology, Royal

North Shore Hospital

R Joffe

Department of

Medicine, University

of Sydney

$G$ A Broe

W Brooks

Correspondence to:

Professor J G L Morris,

Department of Neurology,

A4B, Westmead Hospital,

Sydney, NSW 2145,

Australia.

Received 19 September 1995 and in revised form

2 and in revised form

Accepted 9 January 1996 eosinophilic intraneuronal cytoplasmic inclusions found in the substantia nigra, locus coeruleus, substantia innominata, and dorsal motor nucleus of the vagus nerve in idiopathic Parkinson's disease. In diffuse Lewy body disease, immunocytochemically similar, widespread Lewy bodies are found in the neocor- tex, in addition to classic Lewy bodies in brain stem neurons. ${ }^{12}$ Diffuse Lewy body disease may have pathological features in common with Alzheimer's disease such as diffuse plaques but neurofibrillary tangles and senile neuritic plaques are absent or sparse in pure diffuse Lewy body disease..$^{1-3}$ Significant neuronal loss in the hippocampus and medial temporal or frontal cortices is not present in pure diffuse Lewy body disease, by contrast with Alzheimer's disease. However, a proportion of cases fulfil the pathological criteria for both Alzheimer's disease and diffuse Lewy body disease. ${ }^{245-8}$ These cases are sometimes referred to as patients with senile dementia of the Lewy body type ${ }^{4}$ or the Lewy body variant of Alzheimer's disease. ${ }^{8}$ Lewy bodies have been found in small numbers in Alzheimer's disease without evidence of parkinsonism ${ }^{910}$ and Parkinson's disease and Alzheimer's disease can coexist. ${ }^{11-13}$

Many patients have been reported with cognitive impairment and psychosis as the dominant presentation of diffuse Lewy body disease. $^{2-471415}$ Extrapyramidal signs were less prominent and occurred later or only after treatment with neuroleptic drugs, although some retrospective studies may have underestimated extrapyramidal signs. ${ }^{2-4}$ There are far fewer patients with a parkinsonian presentation. ${ }^{2} 3$ 16-18

Diffuse Lewy body disease presents a broad clinical range that is difficult to diagnose confidently during life. The clinical features overlap with both Alzheimer's disease and Parkinson's disease. There is evidence that diffuse Lewy body disease is one of the most common causes of dementia after Alzheimer's disease. ${ }^{41920}$ To further clarify the syndrome, we present nine patients with pathologically confirmed diffuse Lewy body disease who lack sufficient additional pathology to diagnose as having Alzheimer's disease. In our experience pure diffuse Lewy body disease is not uncommon in patients with onset of prominent parkinsonism before 70 years of age who develop dementia, sometimes after many years. We speculate on the relation between diffuse Lewy body disease and Parkinson's disease and the possible progression of the pathological changes as manifested clinically during life.

\section{Methods}

The cases were collected from brains received by the New South Wales Parkinson's Syndrome Brain Bank (seven of 48) and the 
Alzheimer's disorder brain bank (two of 43) coming for postmortem examination between 1990 and 1994. All of the brains were examined by neuropathologists (GMH and DAMcR). They had been prospectively studied by neurologists or psychiatrists from presentation until death, with detailed records of history, and physical and mental examination. Patients 1 and 7 had detailed serial neuropsychological assessments. The notes of the patients in whom there was no neuropsychologist's examination during life were retrospectively reviewed for further comment by the neuropsychologist (WGJR). Routine haematology, biochemistry, and radiology were performed for treatable causes of dementia.

\section{PATHOLOGICAL DIAGNOSIS}

Necropsies were performed with consent and the study was approved by the human ethics committees of the Universities of Sydney and New South Wales. Brains were collected, weighed, and immersion fixed in $15 \%$ buffered formalin for two weeks. The brainstem was removed from the cerebrum and detached from the cerebellum. The cerebrum and brainstem were embedded in 3\% agar and sectioned at $3 \mathrm{~mm}$ in the coronal and transverse planes respectively. Macroscopic examination of the cut brain slices disclosed that the substantia nigra and locus coeruleus were depigmented in all cases. Cerebral cortical blocks were taken of the superior frontal, ante-

Table 1 Clinical features

\begin{tabular}{|c|c|c|c|c|c|c|c|c|c|}
\hline & \multicolumn{9}{|l|}{ Case } \\
\hline & 1 & 2 & 3 & 4 & 5 & 6 & 7 & 8 & 9 \\
\hline Sex & $\mathrm{F}$ & $\mathbf{M}$ & $\mathbf{M}$ & $\mathbf{M}$ & $\mathbf{M}$ & $\mathbf{M}$ & $\mathbf{M}$ & $\mathbf{M}$ & $\mathbf{F}$ \\
\hline Age at onset (y) & 61 & 58 & 59 & 65 & 65 & 54 & 69 & 66 & 59 \\
\hline Age at death (y) & 72 & 71 & 73 & 73 & 74 & 60 & 75 & 74 & 61 \\
\hline Duration (y) & 11 & 13 & 14 & 8 & 9 & 6 & 6 & 9 & 1.5 \\
\hline 1st symptom & B & B & B & B & B & $\downarrow \mathbf{M}$ & Dys & $\downarrow M$ & $\downarrow M$ \\
\hline Rest tremor & + & + & - & + & + & + & - & - & + \\
\hline Bradykinesia & ++ & ++ & ++ & ++ & ++ & + & + & ++ & ++ \\
\hline Rigidity & ++ & ++ & ++ & ++ & ++ & + & + & ++ & ++ \\
\hline Falling & + & + & - & - & + & - & - & - & + \\
\hline Eye movement & $\mathbf{N}$ & $\downarrow$ & & $\downarrow$ & $\downarrow$ & $\mathrm{N}$ & $\mathbf{N}$ & $\downarrow$ & $\mathbf{N}$ \\
\hline Postural $\downarrow$ BP & - & + & + & - & - & - & - & - & + \\
\hline Prim reflex & - & - & - & - & + & + & - & + & - \\
\hline LD Response & + & ++ & + & ++ & ++ & & & & SL \\
\hline Dyskinsia & - & ++ & - & - & + & & & & - \\
\hline End of dose & + & ++ & - & $\bar{D}$ & + & & & & - \\
\hline Other & Ant & $\mathrm{U}$ & & Ble & $\mathrm{U}$ & Myo & & $\mathrm{U}$ & $\mathrm{U}$ \\
\hline
\end{tabular}

Age of onset was based on history. SL = Slight; $+=$ mild; $++=$ moderate; $-=$ absent; $\mathrm{N}=$ normal; $U=$ urinary frequency or incontinence; $\downarrow=$ reduced; $B=$ bradykinesia; $M=$ memory dys $=$ dysphasia; prim = primitive; $\mathrm{LD}=$ levodopa; ant $=$ anterocollis; ble = blephorospasm; myo $=$ myoclonus.

Table 2 Neuropsychiatric features

\begin{tabular}{|c|c|c|c|c|c|c|c|c|c|}
\hline & \multicolumn{9}{|c|}{ Case } \\
\hline & 1 & 2 & 3 & 4 & 5 & 6 & 7 & 8 & 9 \\
\hline Memory $\downarrow$ & + & + & + & + & + & + & + & + & + \\
\hline Dysphasia & + & - & + & - & + & + & + & + & + \\
\hline Apraxia & + & & & + & + & + & + & + & + \\
\hline $\begin{array}{l}\text { Spatial } \\
\text { disorientation }\end{array}$ & + & & + & + & + & + & + & + & - \\
\hline Agnosia & + & & & + & + & - & + & & + \\
\hline Depression & + & + & + & + & - & + & - & + & + \\
\hline Hallucinations & + & + & + & + & + & - & + & + & - \\
\hline $\begin{array}{l}\text { Episodic } \\
\text { confusion } \\
\text { Other }\end{array}$ & & $\begin{array}{l}\text { Dis } \\
\uparrow \mathrm{Lib}\end{array}$ & + & + & + & Agg & & + & $\begin{array}{l}\text { Dis } \\
\text { Apa }\end{array}$ \\
\hline
\end{tabular}

$+=$ Present; $-=$ absent; $\downarrow=$ decreased; $\uparrow=$ increased; dis $=$ disinhibition; lib $=$ libido; agg = aggression; apa $=$ apathy rior cingulate, primary motor, occipital, inferior temporal, and hippocampal cortices. Subcortical blocks included the caudate and putamen, globus pallidus, thalamus, subthalamus, hypothalamus and mammillary bodies, pons, and medulla oblongata, as well as the cerebellar vermis and dentate nucleus. Blocks were paraffin embedded, cut at $10 \mu \mathrm{m}$, and successive serial sections stained with haematoxylin and eosin, cresyl violet and luxol fast blue, modified Bielschowsky silver, ubiquitin immunohistochemistry and cresyl violet, and tau immunohistochemistry and cresyl violet. Garvey's modification of the Bielschowsky silver stain $^{21}$ was used to quantify plaque and tangle pathology in 10 randomly chosen, high power $(\times 200)$ microscopic fields in the cingulate and parahippocampal gyri. Ubiquitin immunohistochemistry was used to quantify cortical Lewy body pathology in 10 randomly chosen, high power $(\times 200)$ microscopic fields in layers 5 and 6 (infragranular layers) of the cingulate and parahippocampal gyri. Haematoxylin and eosin staining was used to assess midbrain cell loss and Lewy body formation. The ubiquitin antiserum was rabbit polyclonal (Sigma No U5379) diluted 1:100, and the tau antibody was mouse monoclonal (Sigma No T5530) diluted 1:10 000, which recognises only abnormally phosphorylated forms of tau. Cortical Lewy bodies were round ubiquitin positive and silver positive but tau negative intraneuronal inclusions, as previously described. ${ }^{22}$ By contrast, neurofibrillary tangles were also positive for tau. Patients with extensive cortical neurofibrillary tangles and diffuse and neuritic plaque formation would have been diagnosed as having Alzheimer's disease. All patients that had cortical Lewy bodies (at least $3 / 200 \times$ field in four out of 10 successive fields along cortical layers 5/6) without substantial tangle formation were diagnosed as having diffuse Lewy body disease.

\section{Results}

CASE REPORTS

Tables 1 and 2 give a summary of the clinical and neuropsychological details.

\section{Case 1}

A 63 year old woman presented with a 16 month history of stiffness and tremor in the right hand, and difficulty writing. On examination there was a mild resting tremor in the right upper limb, moderate rigidity in the right limbs and neck, and a mild rigidity on the left. Speech was soft and facial expression was mildly reduced. Arm swing was absent on the right; gait and posture were otherwise normal. Balance was mildly impaired. Neuropsychological assessment was within normal limits apart from a borderline visual memory deficit. She improved slightly with bromocriptine but after two years on $22.5 \mathrm{mg}$ per day, developed acute episodes of confusion and complex, formed visual hallucinations. She improved physically and mentally after changing to levodopa-carbidopa $(300 / 75 \mathrm{mg} /$ 
day). At three years abnormalities in her neuropsychological testing were insufficient to diagnose dementia. A significant deterioration had occurred in auditory verbal learning and simple and choice reaction times. At four years her disease was becoming more symmetric and she complained of mild end of dose failure. Depression was treated with mianserin. At five years vivid visual hallucinations and illusions were noted again: silent people moved through her home and the smooth white branch of a eucalyptus tree became the thigh of a nymph. She was alert and could give lucid explanations of her hallucinations. Her auditory verbal and visual memory and simple and choice reaction times were declining. At five years she developed severe anterocollis, a pronounced deterioration in mental state, and falling. At seven years she was disoriented, aphasic, perseverating, severely rigid and bradykinetic, extremely flexed in her cervical and thoracic spine, and confined to a chair or bed. She died with cachexia and pneumonia 11 years after her first symptoms.

\section{Case 2}

A 60 year old man presented with a three year history of fine postural tremor and loss of dexterity in his left hand. There was an intercurrent history of depression treated with doxepine. He had pronounced bradykinesia, mild rigidity, both more prominent on the left, and a resting tremor of his left leg. Ocular pursuit of a target was slowed. He responded well to levodopa-carbidopa ( $400 / 40 \mathrm{mg} /$ day). Three years later he had moderate rigidity in all limbs and moderate left sided dyskinesia. Six years after presentation he was becoming dependent on his family in activities of daily living and developed on-off symptoms, freezing, and a shuffling gait. Dyskinesia became more severe. There was a minor abnormality in eye movements and pursuit was defective. Posture was slightly flexed. Visual hallucinations of a threatening person in his room occurred at night. At nine years he was overactive and disinhibited, making crude and inappropriate remarks to friends and strangers. Libido was excessive and required treatment. He had frequent falls and was posturally hypotensive. At 13 years complex visual hallucinations, overactivity, and disinhibition were a severe problem. He was institutionalised and died five months later.

\section{Case 3}

A 61 year old man presented with a two year history of general slowing in activities of daily living and soft speech. Gait was occasionally shuffling. On examination he was mentally alert, had a masked face, was mildly stooped, and swung his arms poorly, left less than right. There was rigidity in his neck and all limbs and bradykinesia in his upper limbs. He was started on levodopa-carbidopa (300/30 $\mathrm{mg} /$ day) with improvement. Five months later he had septicaemia with pronounced hypotension. Mild cognitive decline and increased rigidity were noted after this. Levodopa-carbidopa was increased gradually to $750 / 75$ $\mathrm{mg} /$ day with little change in his parkinsonism. Depression required doxepine. At four years episodic confusion was noted and bradykinesia was greater on the left. No tremor was seen. At five years pronounced postural hypotension required treatment. By six years confusion, disorientation, and anxiety were increasing problems. He had visual hallucinations nine years after presentation and was moderately demented. He died 14 years after the onset of his symptoms from bronchopneumonia.

\section{Case 4}

A 70 year old man had been diagnosed with Parkinson's disease with bradykinesia and rigidity five years previously. $\mathrm{He}$ had responded poorly to low dose levodopa-carbidopa. He was severely akinetic, expressionless, open mouthed, and drooled saliva. His voice was monotonous and there was slight blepharospasm. There was a minor abnormality of eye movement with broken pursuit. There was moderate axial and limb rigidity and slow fine movements of the fingers. Minimal tremor was noted on one occasion. There was mild cognitive impairment. He responded to increased levodopa-carbidopa. Six years after diagnosis, the addition of benzhexol caused acute frank confusion. Dementia was more definite and confusion continued to occur intermittently. Depression was noted. The next year he had delusions and visual hallucinations, imagining there were men in his house talking to him. He required help with dressing and poured milk into the sugar bowl at breakfast. He continued to decline physically and mentally, dying eight years after first diagnosis.

\section{Case 5}

A 66 year old man presented because his game of croquet had become less skilful and his gait had changed. He was subjectively a little forgetful. On examination he was of superior ability in cognitive tests. His face was slightly lacking in expression and he was bradykinetic. Arm swing was poor. There was mild rigidity, left more than right. A year later he was slightly stooped, he carried both arms in a flexed posture, and did not swing his left arm, which had a slight resting and postural tremor. Rigidity and impaired finger movements were more pronounced on the left. He was started on levodopa-carbidopa and responded well. After 19 months he reported end of dose failure. One year later cognitive tests showed that his responses were average rather than superior. The next year he started falling, had pronounced bradykinesia, and mild to moderate rigidity bilaterally. He responded to increased medication but developed dyskinesia. He had days when he was intermittently unreliable, confused, and disoriented. He exhibited visual agnosia and was unable to tell his razor from his watch. Conversation was rambling and repetitive. Five years after presentation he did not recognise his wife at times, he had nocturnal urinary incontinence, and he was disorientated within his house. $\mathrm{He}$ was increasingly apraxic when dressing and cleaning himself. 
He had hallucinations that the house was full of people. Grasp reflexes were present. Eye movements were normal until late in the disease, when smooth pursuit was broken but a full range of movement was achieved. Later that year he fractured the neck of a femur and was institutionalised. $\mathrm{He}$ rarely spoke and made little sense when he did. He died two years later.

Case 6

A 58 year old man presented with a four year history of memory impairment which deteriorated after a myocardial infarct. He performed very poorly on cognitive tests of new learning and memory retention. He had word finding difficulties. He was disoriented within his neighbourhood. He was noted to have a mildly masked face and positive glabellar tap. $\mathrm{He}$ also had brief myoclonic jerks in his right arm. An EEG was considerably slowed bilaterally with mixed $8 \mathrm{~Hz}$ alpha, $4-6 \mathrm{~Hz}$ theta and occasional bursts of $2-3 \mathrm{~Hz}$ delta activity. One year later he was depressed. He had mild axial rigidity, reduced rapid finger movements, and bilateral grasp reflexes. Three months later he was more bradykinetic and mild rigidity was noted in all limbs. His posture was increasingly stooped and he had dressing and constructional apraxia. Later that year he was constantly confused and became aggressive, incontinent of urine nocturnally, and required constant supervision. He was given thioridazine ( $20 \mathrm{mg}$ per day). He became moderately rigid, bradykinetic, and much more stooped. A resting tremor was noted in his right upper limb. His face was masked and speech was monotonous. Rapid movements were extremely poor bilaterally and complicated by apraxia. He was institutionalised and died suddenly of myocardial infarction several weeks later.

\section{Case 7}

A 74 year old man had retired four years earlier from accountancy as he found the new tax laws too difficult to learn. His memory gradually declined and he presented to a psychiatrist with terrifying visual hallucinations of insects, animals, and people. At neuropsychological assessment he was alert and oriented. His attention and concentration were well sustained. His language was characterised by circumlocutions and word finding difficulties. Verbal fluency was impaired. New learning and retention were intact and there was little other evidence of impaired cognitive function apart from some mild deficits in conceptual abilities and problem solving. Pimozide was of partial help. Follow up neuropsychological assessment nine months later showed a deterioration in all aspects of cognitive functioning. Memory and new learning were significantly compromised. Judgement, reasoning, visuoconstructive and spatial abilities, problem solving, abstraction, and conceptual skills were grossly impaired. $\mathrm{He}$ became disoriented within his home and proposed to his wife. He wandered off and became lost. He required help with dressing and bathing. He was insti- tutionalised and developed an acute confusional state in the presence of pneumonia and sepsis. A shuffling gait, rigidity, and dysphagia were first noted at this time. It was suspected that neuroleptic use contributed to these signs. $\mathrm{He}$ died seven weeks after leaving home, of bronchopneumonia and multifocal pulmonary infarction.

Case 8

A 69 year old man became convinced that TV commentators were in his home and he was referred to a psychiatrist. He had been treated for anxiety since the age of 44 . There had been a slow mental decline in the previous three years. He failed to care for his appearance and was apathetic and withdrawn. One year later a stroke was suspected because of a sudden onset of shuffling gait, unsteadiness, and facial droop but this was not confirmed on CT. The next year he re-presented with acute delirium and hallucinations that the TV presenters were in his home, problems thought to be due to excessive and irregular use of benzodiazepines, phenothiazine, and mianserin. All drugs apart from thioridazine $(75 \mathrm{mg} /$ day $)$ were withdrawn. The delerium and hallucinations improved but he remained confused, disoriented, disinhibited, dysphasic, and apraxic. $\mathrm{He}$ was occasionally incontinent of urine. He had a pronounced reduction in vertical gaze and convergence. Glabellar tap, jaw jerk, and grasp were all positive. Tone was much increased, on the left more than right. Posture was very flexed. His gait was festinating and arm swing was absent. He was unable to walk three years later and died nine years after the onset of symptoms, emaciated and with bronchopneumonia.

\section{Case 9}

This case has been reported previously. ${ }^{23}$ A 59 year old woman became forgetful and a few months later could no longer concentrate to add figures. Her handwriting became a childish scrawl and she was tearful and emotional. On examination she was akinetic with a masked face had micrographia, and required help to get out of bed. She had a mildly exaggerated physiological tremor. There was mild rigidity bilaterally. Her left arm did not swing but her gait was reasonable. Levodopa-carbidopa $(750 / 187.5 \mathrm{mg} /$ day) was of little help. Six months later her speech was unclear and she forgot conversations mid-sentence. There was urinary frequency and urgency. Her posture was flexed and her gait was shuffling. A mild resting tremor was noted intermittently in her left arm. She developed postural hypotension and mild dysphasia. She required increasing help with whole body movements from chair or bed. Eleven months after presentation she often did not recognise her husband and had been admitted to a nursing home. She died two months later of pneumonia.

\section{NEUROPATHOLOGY}

All cases had pathologically confirmed idiopathic Parkinson's disease at necropsy with cell loss and Lewy body formation in the mid- 
Table 3 Neuropathological findings

\begin{tabular}{|c|c|c|c|c|c|c|c|}
\hline \multirow[b]{3}{*}{ Case } & \multirow{2}{*}{\multicolumn{3}{|c|}{ Anterior cingulate }} & \multirow{2}{*}{\multicolumn{2}{|c|}{ Parahippocampus }} & \multicolumn{2}{|c|}{ Midbrain } \\
\hline & & & & & & \multirow[b]{2}{*}{$L B$} & \multirow{2}{*}{$\begin{array}{l}S N \\
\text { Cell } \\
\text { loss }\end{array}$} \\
\hline & Plaque & Tangles & $L B$ & Plaque & Tangles & & \\
\hline $\begin{array}{l}1 \\
2 \\
3 \\
4 \\
5 \\
6 \\
7 \\
8 \\
8 \\
9\end{array}$ & $\begin{array}{l}5-15 \\
0 \\
1-3 \\
1-2 \\
2-6 \\
2-10 \\
1-2 \\
1-2 \\
0\end{array}$ & $\begin{array}{l}0-1 \\
0-1 \\
0 \\
0 \\
0 \\
0 \\
0-1 \\
0 \\
0\end{array}$ & $\begin{array}{l}4-9 \\
1-4 \\
1-4 \\
1-7 \\
0-4 \\
2-6 \\
0-4 \\
1-4 \\
0-4\end{array}$ & $\begin{array}{c}10-20 \\
0 \\
2-3 \\
10-20 \\
1-5 \\
5-10 \\
15 \\
10-20 \\
1-2\end{array}$ & $\begin{array}{l}0-1 \\
0-1 \\
0 \\
0-1 \\
0 \\
0 \\
0-1 \\
0-1 \\
0\end{array}$ & $\begin{array}{l}2-4 \\
3-8 \\
2-5 \\
3-6 \\
4-6 \\
3-6 \\
0-4 \\
1-4 \\
0-4\end{array}$ & $\begin{array}{l}++++ \\
+++ \\
++++ \\
++++ \\
++++ \\
+++ \\
++++ \\
++++ \\
+++\end{array}$ \\
\hline
\end{tabular}

Ranges of the number of diffuse, senile plaques, neurofibrillary tangles, and Lewy bodies (LB) are given for each of 10 randomly chosen, high power $(x 200)$ microscopic fields in the areas designated. $\mathrm{SN}=$ substantia nigra $;+++=$ marked $;++++=$ extreme.

brain substantia nigra. In addition, widespread cortical Lewy bodies (at least $3 / \times 200$ field in four out of 10 successive fields along cortical layer 5/6) were present without substantial neurofibrillary tangle pathology. Thus according to current diagnostic criteria, ${ }^{12425}$ these cases fulfilled the criteria for diffuse Lewy body disease without coexisting Alzheimer's disease. Table 3 gives a summary of the neuropathological findings. Patient 9 was considered initially to have sufficient additional pathology for a diagnosis of co-existent Alzheimer's disease, but on current criteria does not now fulfil the requirements for this. None of the cases described had the spongiform change in the temporal lobes found by Hansen et al. ${ }^{8}$ Patient 7 had a small $(0.7 \times 0.4$ $\times 0.3 \mathrm{~cm}$ ) lacunar infarct in the right internal capsule. All other patients were free of vascular disease.

\section{CLINICAL FEATURES}

There were seven men and two women. Five patients presented with a parkinsonian syndrome, two with dementia, and two with both dementia and parkinsonism. Their mean age of onset was 62 (range 54 to 69) years. This did not differ significantly according to whether the presentation was of parkinsonism or of dementia. The duration of illness from historical onset until death was, however, shorter in those patients with dementia (mean 5.6 years) than in those presenting with parkinsonism (mean 11.0 years).

\section{Parkinsonism with later dementia}

Five patients presented with symptoms due to bradykinesia. They had moderate bradykinesia and rigidity. Resting tremor was noted at some time in four of the five patients but was mild and infrequent. The signs were noted to be asymmetric in four of the five patients initially. All patients developed a parkinsonian gait. Falling occurred in three of the five patients on average seven years after presentation (range five to nine years). Ocular movements were mildly impaired in three patients, with poor pursuit rather than pronounced restriction in the range of eye movement. Postural hypotension was symptomatic in two patients six and eight years after presentation. Grasp reflexes occurred in one patient five years after diagnosis when dementia was present. All five patients responded to levodopa, three well, developing end of dose failure and dyskinesia. Dementia first became apparent 0.5 to 4.5 years after presentation (mean three years) in these five patients. Hallucinations preceded other symptoms of dementia in one patient by six months. In four patients hallucinations developed after the onset of mental impairment. The mean duration from presentation to hallucinations was six (range $2 \cdot 5-9$ ) years.

\section{Dementia at presentation}

Two patients presented with both parkinsonism and dementia. Both had a slight resting tremor and mild to moderate bradykinesia and rigidity. Only one was treated with levodopa, with little response. Two patients presented with neuropsychiatric features alone. They developed parkinsonism later in their illness, one six years and one three years after treatment with phenothiazine. Tremor was not present in these two patients. All patients developed a gait disturbance. Falling occurred in the very late stages. Vertical gaze and convergence were very reduced in one patient. Urinary symptoms were present in two patients. Myoclonus occurred unilaterally in one patient. Delusions and visual hallucinations were the presenting features of one of these four patients. Grasp reflexes and positive jaw jerks were found in two patients.

\section{NEUROPSYCHOLOGICAL FEATURES}

All patients had a rapid and generalised decline in their cognitive functioning. Four of the nine patients $(1,3,4,9)$ presented initially with deficits consistent with a subcortical dementia characterised by mild early memory impairment and slowing of information processing. Three $(5,6,8)$ had early memory impairment, one had behavioural signs of frontal lobe dysfunction (patient 2), and one (patient 7) presented with prominent early language problems consistent with dominant temporal lobe dysfunction. All progressed to more widespread cognitive deficits consistent with extensive cortical dysfunction.

Memory impairment was among the earliest signs of dementia in eight patients. Apraxia was prominent in seven patients and five patients developed agnosia. Language impairment developed in seven patients. Depression was present in seven patients often early in the disorder. Four patients required treatment with antidepressant medication.

Complex visual hallucinations occurred in seven patients. Hallucinations occurred in three (patients 1, 2, 7) at times when the patient was otherwise alert but also at times of delerium (patients 1 and 8 related to medication), and at times when cognitive decline was pronounced and confusion was constant (patients 2, 3, 4, 5).

\section{Discussion}

There has been increased recognition of diffuse Lewy body disease since the first report in $1961,{ }^{26}$ with increasing awareness of the condition and improved neuropathology. This necropsy sample was restricted to patients 
thought to have Parkinson's disease or Alzheimer's disease but the sample size is not sufficiently large to give a true perspective of the incidence of diffuse Lewy body disease. The recruitment of cases into the New South Wales Parkinson's syndrome brain bank is considered to be biased towards unusual cases of Parkinson's disease, due to the greater interest of the referring neurologist in obtaining the true diagnosis, although all members of the Parkinson's syndrome society of New South Wales and the patients attending Parkinson's disease clinics are requested to consider donation of their brains. However, this study and those of Yoshimura ${ }^{27}$ and Sugiyama $e t a l^{8}$ suggest that diffuse Lewy body disease may be a common cause of dementia in patients presenting as Parkinson's disease.

\section{PATIENTS WITH PARKINSONISM BUT NO}

DEMENTIA AT PRESENTATION

Parkinsonism without dementia at presentation is less common in previous reports of diffuse Lewy body disease, ${ }^{2-48}$ amounting to about $20 \%$ of cases, compared with dementia at presentation. A parkinsonian presentation seems to be more common in pure diffuse Lewy body disease ${ }^{2}$ than in the Lewy body variant of Alzheimer's disease. In this series, resting tremor is often present but is mild and infrequent. Tremor is the least likely of the signs in the parkinsonian diagnostic triad to be reported in diffuse Lewy body disease. ${ }^{2} 381718$ A good response to levodopa is recorded on rare occasions ${ }^{2371618}$ and no response on others. ${ }^{1729}$ Our findings are similar to those of Byrne et $a l^{16}$ who found that $6 / 15$ cases presented with parkinsonism alone and that $10 / 11$ cases had a definite response to levodopa. When a parkinsonian syndrome was prominent with little dementia, a response to levodopa was more likely, perhaps because the levodopa dose was not restricted by neuropsychiatric side effects or because the disease was confined to the substantia nigra initially. It is increasingly recognised that in parkinsonian syndromes a poor response to levodopa often indicates more widespread pathology than is found in idiopathic Parkinson's disease.

All patients later developed widespread cognitive problems consistent with a cortical dementia. However, early in the disease four patients with parkinsonian signs at presentation had evidence of a subcortical dementia alone, a form of dementia typical of idiopathic Parkinson's disease, ${ }^{30}$ before developing more widespread signs of cortical dysfunction.

\section{Patients with dementia at presentation}

The most typical presentation of diffuse Lewy body disease - early neuropsychiatric features and dementia, and mild, late parkinsonian signs $^{2-48}$ - occurred in two of our patients. The presence of Alzheimer's type neuritic pathology in some patients with diffuse Lewy body disease with dementia at presentation in previous reports may give a falsely high incidence of early and dominant dementia. ${ }^{8141631}$ Another two patients had dementia at presentation and definite features of parkinsonism, much more than would be found in Alzheimer's disease alone. This presentation is similar to that in patients with pathologically established coexistent idiopathic Parkinson's disease and Alzheimer's disease (personal unpublished observation).

Impaired memory and inability to learn new material were the presenting features in three of four patients with dementia at presentation. One patient presented with language dysfunction and hallucinations. These features suggest that the disease started in the cerebral cortex and only later did the brain stem become involved. The use of phenothiazine in those with early dementia certainly worsened the parkinsonian features in one and precipitated parkinsonian features in two. The precipitation of parkinsonian signs by phenothiazines in patients with dementia and diffuse Lewy body disease are well documented. ${ }^{32}$

Patients presenting with dementia had a more rapidly progressive course, as has been found previously in cases of parkinsonism and early dementia, diffuse Lewy body disease presenting with dementia or Alzheimer's disease with parkinsonian features. ${ }^{33-35}$ Conversely, patients without dementia at onset had a mean duration of disease closer to that of pathologically established idiopathic Parkinson's disease $(13 \cdot 1$ years $) .^{13}$

\section{Neuropsychological features of the nine patients}

Depression is commonly described in diffuse Lewy body disease $e^{71536}$ and was present in seven $(78 \%)$ patients in our series. This is a much higher incidence than reported previously in clinically diagnosed Parkinson's disease $^{37}$ or Alzheimer's disease. ${ }^{15}$ Disinhibition occurred in two patients and apathy and anhedonia in one of these suggesting frontal lobe dysfunction. Aphasia, apraxia, spatial disorientation, and agnosias were prominent once dementia developed. These features are consistent with a cortical dementia. The pathological lesion causing the dementia is unknown.

Hallucinations were generally vivid and visual consisting of people, but sometimes animals, in the home. They occurred at times when the patient was otherwise lucid and had insight into their nature but also at times of delerium, sometimes exacerbated by drugs, and also when the patient was demented. Hallucinations were common in diffuse Lewy body disease in previous reports. ${ }^{14-1738}$ In this series hallucinations occurred in seven $(78 \%)$ patients. In only one patient were they an early feature, unlike some previous reports. ${ }^{79163538}$ They were commonly an intermediate feature in our series in those who presented with Parkinson's disease, occurring on average five years after presentation. Paranoia and aggression were far less common. These features were managed by reducing antiparkinsonian medication, if used. Episodic confusion occurred late in the disease in four patients. This is said to be an indicator of diffuse Lewy body disease in the absence of a clear explanation of deterioration. ${ }^{15} 16$ It may lead to a suspicion of cerebrovascular disease but this has not 
been found in our patients. Such day to day fluctuations in cognition are rare in idiopathic Parkinson's disease ${ }^{13}$ and uncommon in Alzheimer's disease. ${ }^{15}$

The relation to idiopathic Parkinson's disease and Alzheimer's disease

The treating neurologists considered that idiopathic Parkinson's disease was the correct diagnosis initially in the five patients without dementia at presentation. Blepharospasm, although uncommon, has been found in both Parkinson's disease ${ }^{13}$ and multisystem atrophy, ${ }^{39}$ as has anterocollis. ${ }^{133940}$ In our experience primitive reflexes are rare in Parkinson's disease without dementia. Falling can occur in any of the parkinsonian syndromes and was not unusually early in most of our patients to suggest a diagnosis other than idiopathic Parkinson's disease. Unexplained falls are reported to be more common in patients with diffuse Lewy body disease than in patients with Alzheimer's disease. ${ }^{15}$ Myoclonus seems to be the most atypical movement disorder shared least with the other syndromes. There are occasional reports of myoclonus in diffuse Lewy body disease ${ }^{36313841}$ and mainly stimulus sensitive distal myoclonus in multisystem atrophy. ${ }^{40}$

It is possible that in the five patients without dementia at presentation, typical idiopathic Parkinson's disease with classic nigral Lewy bodies evolved into diffuse Lewy body disease as the cortical Lewy bodies later appeared, possibly hand in hand with the worsening dementia. This supports a continuum of the disease process from Parkinson's disease to diffuse Lewy body disease. The occasional Lewy body is found in the neocortex of most patients with Parkinson's disease, further linking the diseases. ${ }^{1328}$ Cases intermediate between Parkinson's disease and diffuse Lewy body disease pathologically (few cortical Lewy bodies, typical idiopathic Parkinson's disease brainstem pathology, ubiquitin positive linear neurites in the CA2 region of the hippocampus) presenting with initial dementia have been reported. ${ }^{42}$

More commonly, diffuse Lewy body disease has intercurrent Alzheimer's disease changes neuropathologically. Patients are often older than patients with pure diffuse Lewy body disease and dementia is more common initially. ${ }^{24162038}$ Extrapyramidal signs are often mild. The mean age of onset in our patients with diffuse Lewy body disease was identical to that in the clinically diagnosed Sydney multicentre study of Parkinson's disease ${ }^{43}$ and in the pathologically diagnosed United Kingdom Parkinson's disease brain bank. ${ }^{13}$ All patients were aged less than 70 years at presentation.

The diagnostic criteria for Alzheimer's disease, diffuse Lewy body disease, and diffuse Lewy body disease with Alzheimer's disease have varied considerably in the past. ${ }^{248384144}$ Although a relation between Alzheimer's disease and diffuse Lewy body disease was thought to exist, ${ }^{8}$ they are now considered to be distinct pathologically. ${ }^{12520}$ We have followed the stricter diagnostic criteria set down by Lippa et al, ${ }^{1}$ which excluded the presence of neurofibrillary tangles and neuritic plaques in pure diffuse Lewy body disease. Clinical criteria to differentiate the two disorders in life have been proposed, with some clear differences existing. ${ }^{45}$ It is possible that diffuse Lewy body disease and idiopathic Parkinson's disease are more closely related, although cortical Lewy bodies in diffuse Lewy body disease have subtle differences from brain stem Lewy bodies $^{78}$ and the aetiology and genetics of both disorders are poorly understood. All of our patients met the pathological criteria of idiopathic Parkinson's disease in addition to the more diffuse cortical Lewy body changes. They had dementia without Alzheimer's type neuritic pathology or evidence of diffuse cerebrovascular disease. Our patients did have mild to moderate numbers of diffuse plaques but lacked cored plaques. This pattern of diffuse plaque pathology without cored plaques or neurofibrillary tangles is common in nondemented elderly people. ${ }^{46}$ Most patients with plaques who are demented tend to have the addition of either cortical Lewy bodies ${ }^{24}$ or cortical neurofibrillary tangles..$^{25}$ It would seem that intracellular inclusions, in addition to extracellular amyloid deposits, are necessary for dementia in most cases.

We suggest that diffuse Lewy body disease may start in different areas, either cortical or subcortical, in different patients, and the pattern of the pathological progression determines the presentation-that is, if the disease commences in the substantia nigra the patient may have dopa responsive Parkinson's disease but with time cortical involvement and dementia develops; whereas if the disease starts in the cerebral cortex then dementia and neuropsychiatric features dominate the picture initially and parkinsonian features may appear later.

\section{Conclusions}

Pure diffuse Lewy body disease may present as indistinguishable from levodopa responsive idiopathic Parkinson's disease. It may be a common cause of dementia in Parkinson's disease, particularly in younger patients. The presentation of dementia with early parkinsonian features should suggest diffuse Lewy body disease but it is clinically difficult to distinguish it from Alzheimer's disease and coexistent idiopathic Parkinson's disease in our experience. The presence of fluctuating confusion, hallucinations, or myoclonus may help in making the diagnosis. The dementia is of cortical type when fully developed. The combination of early dementia and parkinsonism has a poor prognosis. Only further pathological study of patients with dementia or parkinsonism without population bias will help clarify the true incidence and importance of diffuse Lewy body disease.

1 Lippa CF, Smith TW, Swearer JM. Alzheimer's disease and Lewy body disease: a comparative clinico-pathological study. Ann Neurol 1994;35:81-8.

2 Kosaka K. Dementia and neuropathology in Lewy body disease. Adv Neurol 1993;60:456-63.

3 Gibb WRG, Luthert PJ, Janota I, Lantos PL. Cortical Lewy body dementia: clinical features and classification. 
f Neurol Neurosurg Psychiatry 1989;52:185-92.

4 Perry RH, Irving D, Blessed G, Fairbairn A, Kerry EK Senile dementia of Lewy body type. A clinically and neuropathologically distinct form of Lewy body dementia in the elderly. $\mathcal{F}$ Neurol Sci 1990;95:119-39.

5 Ince $\mathrm{P}$, Irving $\mathrm{D}$, MacArther F, Perry RH. Quantitative neuropathological study of Alzheimer-type pathology in the hippocampus: comparison of senile dementia Alzheimer type, senile dementio of Lewy body Parkinson's disease and non demented elderly control patients. ₹ Neurol Sci 1991;106:142-52.

6 Forno LS, Barbour PJ, Norville RI. Presenile dementia with Lewy bodies and neurofibrillary tangles. Arch Neurol with Lewy bodies

7 Gibb WG, Esiri MM, Lees AJ. Clinical and pathological features of diffuse cortical Lewy body disease (Lewy body dementia). Brain 1985;110:1131-53

8 Hansen LA, Salmon D, Galasko D, et al. The Lewy body variant of Alzheimer's disease: a clinical and pathological entity. Neurology 1990;40:1-8

9 Gibb WRG, Mountjoy CQ, Mann DMA, Lees AJ. A pathological study between Lewy body disease and Alzheimer's disease. $\mathcal{F}$ Neurol Neurosurg Psychiatry 1989; 52:701-8.

10 Rosenblum WI, Ghatak NR. Lewy bodies in the presence of Alzheimer's disease. Arch Neurol 1979;36:170-1.

11 Boller E, Mizutani T, Roessmann U, Gambetti P. Parkinson's disease, dementia and Alzheimer's disease. Ann Neurol 1980;7:329-35.

12 Vermersch P. Dementia in Parkinson's disease. Ann Neurol 1993:33:445-50.

13 Hughes AJ, Daniel SE, Blankson S, Lees AJ. A clinicopathologic study of 100 cases of Parkinson's disease. Arch Neurol 1993;50:140-8.

14 Kuzuhara S, Yoshimura M. Clinical and neuropathological aspects of diffuse Lewy body disease in the elderly. $A d v$ Neurol 1993;60:464-9.

15 McKeith IG, Perry RH, Fairbairn AF, Jabeen S, Perry EK. Operational criteria for senile dementia of Lewy body type (SDLT). Psychol Med 1992;22:911-22.

16 Byrne EJ, Lennox G, Lowe J, Godwin-Austen RB. Diffuse Lewy body disease: clinical features in 15 cases. I Neurol Neurosurg Psychiatry 1989;52:709-17.

17 Mark MH, Sage JI, Dickson DW, Schwarz KO, Duvoisin RC. Levodopa-nonresponsive Lewy body parkinsonism: clinico-pathologic study of 2 cases. Neurology 1992;42: 1323-7.

18 Louis ED, Goldman JE, Powers JM, Fahn S. Parkinsonian features of eight pathologically diagnosed cases of diffuse Lewy body disease. Mov Disord 1995;10:188-94.

19 Lennox G, Lowe J, Morrell K, Landon M, Mayer RJ. Antiubiquitin immunocytochemistry is more sensitive than conventional techniques in the detection of diffuse Lewy body disease. I Neurol Neurosurg Psychiatry 1989;52: 67-71.

20 Dickson DW, Ruan D, Crystal H, et al. Hippocampal degeneration differentiates diffuse Lewy body disease degeneration (DLBD) from Alzheimer's disease: light and electron microscopic immunocytochemistry of CA2-3 neurites specific to DLBD. Neurology $1991 ; 41: 1402-9$.

21 Halliday GM, Flowers D, Baum L. Analysis of staining methods for different cortical plaques in Alzheimer's disease. Acta Neuropathol (Berl) 1994;87:174-86.

22 Love S, Nicholl JAR. Comparison of modified Bielschowsky silver impregnation and anti-ubiquitin immunostaining of cortical and nigral Lewy bodies. Neuropathol Appl Neurobiol 1992;18:585-92.

23 Pamphlett R, Morris J, Leicester J. A woman with dementia and Parkinsonism unresponsive to levodopa. Med $\mathcal{f}$ Aust 1988;149:490-4.

24 Hansen LA, Masliah E, Galasko D, Terry RD. Plaque-only Alzheimer disease is usually the Lewy body variant, and vice versa. $\mathcal{F}$ Neuropathol Exp Neurol 1993;52:648-54.
25 Mirra S, Heyman A, McKeel D, et al. The consortium to establish a registry for Alzheimer's disease (CERAD). Part II. Standardisation of the neuropathological assessment of Alzheimer's disease. Neurology 1991;41:479-86.

26 Okazaki H, Lipkin LE, Aronson SM. Diffuse intracytoplasmic ganglionic inclusions (Lewy type) associated with progressive dementia and quadriparesis in flexion. $f$ Neuropathol Exp Neurol 1961;20:237-44.

27 Yoshimura $M$. Pathological basis for dementia in elderly patients with idiopathic Parkinson's disease. Eur Neurol 1988;28(suppl 1):29-35.

28 Sugiyama $H$, Hainfellner JA, Yoshimura $M$, Budka $H$. Neocortical changes in Parkinson's disease revisited. Clin Neuropathol 1994;13:55-9.

29 Eggertson DE, Sima AAF. Dementia with cerebral Lewy bodies. Arch Neurol 1986;43:524-7.

30 Cummings JL, Benson DF. Dementia. A clinical approach. 2nd ed. Oxford: Butterworth-Heinemann 1992:9-10.

31 Förstl H, Burns A, Luthert P, Cairns N, Levy R. A Lewy body variant of Alzheimer's disease. Clinical and pathological findings. $\mathrm{Br} \mathcal{F}$ Psychol 1993;162:385-92.

32 McKeith I, Fairbairn A, Perry R, Thompson P, Perry E. Neuroleptic sensitivity in patients with senile dementia of Lewy body type. BMF 1992;305:673-8.

33 McKeith IG, Fairbairn AF, Perry RH, Thompson P. The clinical diagnosis and misdiagnosis of senile dementia of clinical diagnosis and misdiagnosis of senile dementia of

34 Hely MA, Morris JGL, Reid WGJ, et al. Age of onset: the major determinate of outcome in Parkinson's disease. Acta Neurol Scand 1995;92:455-65.

35 Stern Y, Mayeux R, Sano M, Hauser WA, Bush T. Predictors of disease cause in patients with probable Alzheimer's disease. Neurology 1987;37:1649-53.

36 Crowe SF, Peppard RF, Borenstein R, Lloyd JH. Diffuse Lewy body disease and progressive dementia in a young woman. $A N Z$ f Psych 1992;26:507-11.

37 Starkstein SE, Preziosi TJ, Bolduc PC, Pobinson RG Prinson's disease $f$ Nerv Ment Dis 1990;178:27-31.

38 Burkhardt CR, Filley CM, Kleinschmidt-DeMasters BK, et al. Diffuse Lewy body disease and dementia. Neurology 1988;38: $1520-8$.

39 Jankovic J, Rhajput AH, Golbe LI, Goodman JC. What is it? Case 1, 1993: parkinsonism, dysautonomia and ophthalmoparesis. Mov Disord 1993;8:525-32.

40 Wenning GK, Ben Shlomo Y, Magalhāes M, Daniel SE Quinn NP. Clinical features and natural history of multiple system atrophy. An analysis of 100 cases. Brain 1994;117:835-45.

41 Crystal HA, Dickson CW, Lizardi JE, Davies P, Wolfson LI. Antimortum diagnosis of diffuse Lewy body disease. Neurology 1990;40:1523-8.

42 Hedera P, Cohen ML, Lerner AJ, Friedland RP. Dementia preceeding motor symptoms in Parkinson's disease. A case study. Neuropsychiatry, Neuropsychology and Behavioural Neurology 1994;7:67-72.

43 Hely MA, Morris JGL, Reid WGJ, et al. The Sydney mult centre study of Parkinson's disease: a randomised prospective five year study comparing low dose prospective five year study comparing low dose Neurol Neurosurg Psychiatry 1994;57:903-10.

44 Khachaturian ZV Diagnosis of Alzheimer's disease. Arch Neurol 1985;42:1097-104.

45 McKeith IG, Fairbairn AF, Bothwell RA, et al. An evaluation of the predictive validity and inter-rater reliability of clinical diagnostic criteria for senile dementia of Lewy body type. Neurology 1994;44:872-7.

46 Armstrong RA. Beta-amyloid deposition in the medial temporal lobe in elderly non-demented brains and in Alzheimer's disease. Dementia 1995;6:121-5.

47 Braak $H$ and Braak E. Neuropathological stageing of Alzheimer-related changes. Acta Neuropathol 1991;82: 239-59. 